\title{
Narrow-line magneto-optical cooling and trapping of strongly magnetic atoms
}

\author{
Andrew J. Berglund ${ }^{1}$, James L. Hanssen ${ }^{1,2}$, and Jabez J. McClelland ${ }^{1}$ \\ ${ }^{1}$ Center for Nanoscale Science and Technology, National Institute of Standards and Technology, Gaithersburg, MD 20899 \\ ${ }^{2}$ Maryland NanoCenter, University of Maryland, College Park, MD 20742
}

(Dated: October 22, 2018)

\begin{abstract}
Laser cooling on weak transitions is a useful technique for reaching ultracold temperatures in atoms with multiple valence electrons. However, for strongly magnetic atoms a conventional narrow-line magneto-optical trap (MOT) is destabilized by competition between optical and magnetic forces. We overcome this difficulty in Er by developing an unusual narrow-line MOT that balances optical and magnetic forces using laser light tuned to the blue side of a narrow $(8 \mathrm{kHz})$ transition. The trap population is spin-polarized with temperatures reaching below $2 \mu \mathrm{K}$. Our results constitute an alternative method for laser cooling on weak transitions, applicable to rare-earth-metal and metastable alkaline earth elements.
\end{abstract}

The extension of laser cooling techniques to weak, narrow-line optical transitions [1, 2] opens new possibilities to reach low temperatures and high phasespace densities for atoms with multiple valence electrons [3]. The main advantage of a kilohertz-linewidth $(\Gamma / 2 \pi \approx 1 \mathrm{kHz})$ transition is the low Doppler temperature, $\hbar \Gamma /\left(2 k_{B}\right)$, which in principle enables Doppler laser cooling to nanokelvin temperatures without evaporative techniques. However, the maximum radiation pressure force that can be applied, $\hbar k \Gamma / 2$, is correspondingly small for a narrow transition and may not even be sufficient to levitate atoms against gravity $(2 \pi / k$ is the optical wavelength). Experimental studies of narrow-line laser cooling have concentrated on the ${ }^{1} \mathrm{~S}_{0}$ to ${ }^{3} \mathrm{P}_{1}$ transitions in $\mathrm{Mg}, \mathrm{Ca}$, and Sr. For Sr [3, 4, [5, 6, 7], optical forces are sufficient to compensate gravity, but the resulting interplay of forces modifies the trap dynamics $[\underline{5}, \underline{6}]$. For $\mathrm{Ca}$ and $\mathrm{Mg}$, optical forces are weaker ( $\Gamma$ is smaller), so that more complicated schemes such as quenched cooling 8, 9], metastable trapping [10], and two-photon cooling 11] are necessary for stable trapping against gravity.

The rare-earth-metal element Er also has multiple valence electrons and narrow optical transitions [12], but in contrast to the alkaline earths, Er also has a large ground state magnetic moment $\left(7 \mu_{B}\right)$ that provides an additional "handle" for manipulation. For the narrow $(\Gamma / 2 \pi=8 \mathrm{kHz})$ transition at $841 \mathrm{~nm}$ in Er, the magnetic force is equal to the maximum radiation pressure force at a field gradient of only $0.3 \mathrm{~T} / \mathrm{m}$. As a result, a magnetooptical trap (MOT) is destabilized if the trapping lasers tend to drive atoms into untrapped magnetic sublevels. In this Letter, we exploit this comparable magnitude of optical and magnetic forces in order to form a narrow-line MOT for Er. In our "strongly magnetic" MOT, confinement is provided by a combination of dissipative optical forces and conservative magnetic forces. The trap is globally stable when the trapping lasers are tuned to the blue side of the (unshifted) atomic resonance. The resulting trap forms at a position of non-zero magnetic field, with a spin-polarized population at temperatures below $2 \mu \mathrm{K}$. In a conventional MOT, on the other hand, confinement forces are purely optical, cooling occurs for red-detuned lasers, and the trap population is (on average) unpolarized at a position of zero magnetic field. The unusual features of our trap are explained by a Doppler cooling model including optical pumping between magnetic sublevels. Our results constitute the lowest temperature achieved for Er atoms and provide an important benchmark in the study of highly magnetic rare-earth-metal elements.

Magnetic forces modify the description of magnetooptical cooling and trapping in a nontrivial way. Consider the $z$ motion of an atom in a magnetic field $B(z)=B^{\prime} z\left(B^{\prime}>0\right)$, together with a circularly polarized traveling-wave laser beam (frequency $\omega_{L}$, wave vector $\vec{k}=k \hat{z}$ with positive or negative $k$ corresponding to the direction of propagation) detuned by $\delta=\omega_{L}-\omega_{A}$ from the atomic transition $\omega_{A}$. We restrict attention to $z>0$ where $B(z)$ is positive. Including the gravitational force $-M g \hat{z}$, the semiclassical position $z$ - and velocity $v_{z}$-dependent force on the atom is [5, [6, 13, 14]

$$
F_{z}\left(z, v_{z}\right)=F_{0}+\frac{\hbar k \Gamma}{2} \frac{s}{1+s+4\left(\delta / \Gamma-z / z_{0}^{ \pm}-k v_{z} / \Gamma\right)^{2}}
$$

where

$$
F_{0}=-g_{g} m_{J} \mu_{B}\left|B^{\prime}\right|-M g \quad, \quad z_{0}^{ \pm}=\frac{\hbar \Gamma}{\mu_{ \pm}^{\prime}\left|B^{\prime}\right|} .
$$

$\mu_{ \pm}^{\prime}=\left( \pm g_{e}+m_{J} \Delta g_{e g}\right) \mu_{B}, g_{g}$ and $g_{e}$ are the Landé factors for the ground and excited states, $\Delta g_{e g}=g_{e}-g_{g}, m_{J}$ is the projection of the angular momentum $J$ along the local magnetic field direction, $s$ is the saturation parameter [14], and \pm corresponds to $\sigma^{ \pm}$polarization.

The force field of Eq. (11) is characterized by the parameter $\mathfrak{F}_{0}=2 F_{0} /(\hbar k \Gamma)$, the ratio of the magnetic and gravitational forces to the light force. For $k<0, \sigma^{-}$ polarization, and red laser detuning $\delta<0$, simultaneous cooling and trapping (i.e., stable equilibrium in both $z$ and $\left.v_{z}\right)$ are achieved for $0<\mathfrak{F}_{0}<s /(1+s) \leq 1$, which also requires $m_{J}<0$. Near equilibrium, the force is approximately $F_{z}\left(z, v_{z}\right) \approx-\kappa\left(z-z_{e q}\right)-\beta v_{z}$ where $z_{e q}$ is 


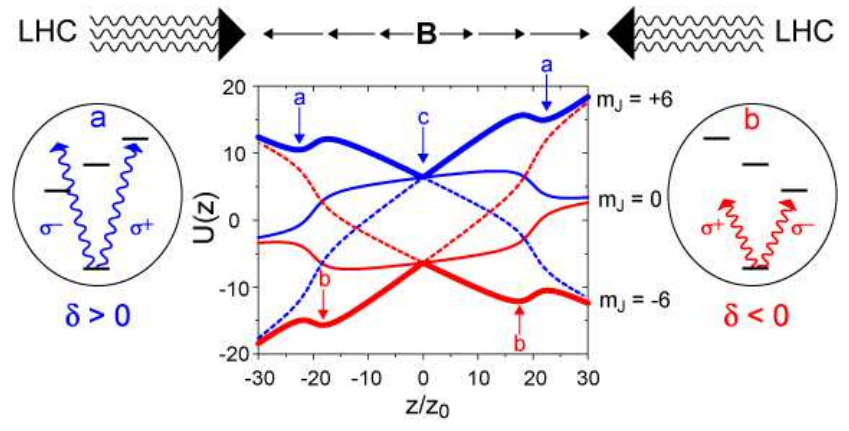

FIG. 1: (Color online) Potential energy for a magnetic atom in a narrow-line MOT. $U(z)$ is in units of $\hbar k \Gamma z_{0} / 2$. At the top, counterpropagating laser beams are labeled by their left-hand circular (LHC) helicity. Curves are shown for an atom with $J=6$, for red or blue detuning and various values of $m_{J}$. For an atom in state $m_{J}$, the curve at higher (lower) energy [blue (red) online] represents the potential for $\delta>0(\delta<0)$. Optical pumping stabilizes the stretched trap states $\left(m_{J}= \pm J\right)$, since blue (red)-detuned trapping lasers tend to drive atoms toward the upper (lower) potential energy curve. Increasing line thickness (from dashed to thin to thick lines) represents increasing population of a state due to optical pumping. Insets a) and b) show the resonant optical transitions at trap minima labeled $a$ and $b$. Here, for $m_{J}=J, \mathfrak{F}_{0}=1 / 2$ in the absence of gravity; the gravitational force is $20 \%$ of the magnetic force.

the trap position, and $\kappa$ and $\beta$ define the spring constant and velocity damping coefficient, respectively. At $z_{e q}$, magnetic forces push the atom away from the origin while optical forces push it back toward the origin, and the red-detuned laser provides Doppler cooling towards $v_{z}=0$. While cooling and trapping may be expected for a red-detuned beam, a similar equilibrium point exists for $\delta>0, \sigma^{+}$polarization, and $k>0$ in the weak-field seeking states with $m_{J}>0$. In this case, magnetic forces pull the atom toward the origin while optical forces push it away and the laser still provides Doppler cooling because it is red-detuned from the Zeeman-shifted transition frequency at the trap position. Therefore, a stable trap forms for appropriately polarized red- or blue-detuned laser light, as long as the direction of laser propagation opposes $F_{0}$ and the radiation pressure is sufficiently large.

Now consider counterpropagating laser beams carrying opposite angular momenta, as in a standard MOT [14]. In this situation, the sign of the magnetic force reverses at $z=0$, and the same circular polarization induces $\sigma$ transitions of opposite sign. As a result, stable equilibria form on either side of the origin, and a conservative magnetic trap also forms at $z=0$ for weak-field seeking $\left(m_{J}>0\right)$ substates. Generalizing Eq. (1) to include the changing sign of the magnetic field and summing the force of each beam, we find the potential energy curves $U(z)=-\int F_{z}\left(z^{\prime}, 0\right) d z^{\prime}$ shown in Fig. 1. For the nonmagnetic $m_{J}=0$ substate with $\delta<0$, the potential energy $U(z)$ reproduces the results in Refs. [5, [6] for a far-
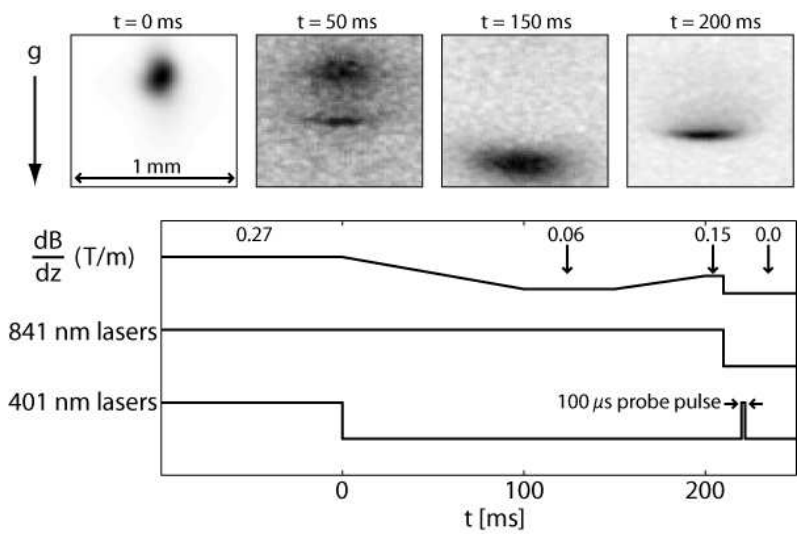

FIG. 2: Cloud images (top) and timing sequence (bottom) during trap loading.

detuned, narrow-line Sr MOT in the presence of gravity. For magnetic states $\left(m_{J} \neq 0\right)$ in the absence of gravity, the potential minima at points labeled $a$ and $b$ exhibit identical values of $\kappa$ and $\beta$. Point $c$ is a purely magnetic trap. Optical pumping populates the "stretched" trap states $\left|m_{J}\right|=J$ in a detuning-dependent way. Bluedetuned trapping lasers become resonant on $\sigma^{+}$transitions and therefore tend to drive atoms to the $m_{J}=+J$ magnetic substate exhibiting a globally stable, $\mathrm{V}$-shaped trap potential. In contrast, red-detuned lasers approach resonance on $\sigma^{-}$transitions tending to drive atoms to the $m_{J}=-J$ substate, with a globally unstable $\Lambda$-shaped potential. We thus expect, and find in practice, that the blue-detuned trap is quite robust, while we were unable to observe any trap in the red-detuned case. Note also that magnetic forces can compensate gravity in this trap, and thus optical forces need not exceed gravitational forces. This trap may therefore be useful for narrowline cooling of metastable, magnetically trapped alkaline earth atoms 10] in cases where $\hbar k \Gamma / 2 \lesssim M g$, or even for cooling on ultranarrow transitions such as the $2.1 \mathrm{~Hz}$ transition in Er [12].

To investigate the cooling limits of this force, we note that near $z_{e q}$ the kinetic energy of an atom increases by twice the photon recoil energy $\left(\hbar^{2} k^{2} / 2 M\right)$ at the equilibrium absorption rate $\mathfrak{F}_{0} \Gamma / 2$, while the energy damping rate is $\beta v_{z}^{2}[13]$. These heating and damping rates are equal for a kinetic energy distribution corresponding to a temperature

$$
T_{e q}=T_{D} \frac{s / 2}{\mathfrak{F}_{0} \sqrt{s / \mathfrak{F}_{0}-1-s}} \quad, \quad T_{D}=\frac{\hbar \Gamma}{2 k_{B}} .
$$

Recalling the stability constraint that $0<\mathfrak{F}_{0}<s /(1+s)$, we find that $T_{e q}$ reaches a minimum of $T_{D}$ for a very dilute, spatially-extended trap, i.e., for $\mathfrak{F}_{0} \ll 1$. However, one is usually interested in creating a compact sample that is not only cold but also dense. Neglecting gravity for simplicity, we find that the $1 \mathrm{D}$ phase-space 

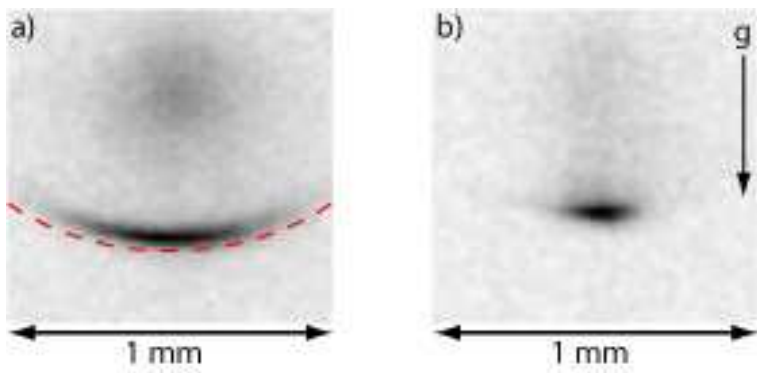

FIG. 3: (Color online) Narrow-line MOT images. a) Cooling and trapping with a single downward propagating beam. Atoms collect along an ellipse of constant magnetic field (dashed line) where they come into resonance with the laser. b) Full 3D narrow-line MOT. In both images, a faint cloud of atoms trapped in the conservative magnetic trap can be seen as well.

density is maximized for $\mathfrak{F}_{0}=1 / 2$. For the $841 \mathrm{~nm}$ transition in Er, the highest phase-space density is predicted to occur at a reasonable magnetic field gradient of $0.15 \mathrm{~T} / \mathrm{m}$ with $1 \mathrm{D}$ kinetic energy distribution corresponding to $T_{e q}=2 T_{D}=380 \mathrm{nK}$.

To observe these dynamics, we modified the apparatus in Refs. [15, 16]. In brief, approximately $10^{5}{ }^{166} \mathrm{Er}$ atoms are trapped and cooled to $150 \mu \mathrm{K}$ in a standard MOT on the bright $(\Gamma / 2 \pi=36 \mathrm{MHz}[17])$ transition at $401 \mathrm{~nm}$. For narrow-line cooling, we use an $841 \mathrm{~nm}$ diode laser locked by a fast servo to a passively stable cavity [18]. Acousto-optic modulators are used for fine control of both the frequency and power. At the MOT position, we use a maximum $841 \mathrm{~nm}$ laser power of $3 \mathrm{~mW}$ distributed into three orthogonal beams with Gaussian waists of $(4.5 \pm 0.05) \mathrm{mm}[19]$. The $401 \mathrm{~nm}$ beams are tilted by $6^{\circ}$ to provide optical access for the $841 \mathrm{~nm}$ beams. Temperature measurements are made by turning off all optical and magnetic fields then imaging fluorescence from the expanding cloud by pulsing on the $401 \mathrm{~nm}$ MOT lasers for $100 \mu \mathrm{s}$.

The narrow-line MOT loading procedure is shown in Fig. 2, Atoms are initially trapped and cooled on the $401 \mathrm{~nm}$ transition (the $841 \mathrm{~nm}$ beams are also on during this period). At time $t=0$, the $401 \mathrm{~nm}$ beams are extinguished leaving only the $841 \mathrm{~nm}$ beams. The field gradient is ramped down over $100 \mathrm{~ms}$ to allow atoms to collect in the narrow-line MOT, then raised to recompress the atomic cloud. Two typical narrow-line MOT fluorescence images are shown in Fig. 3. In image a), we used a single blue-detuned $(\delta / 2 \pi \approx 1 \mathrm{MHz})$ beam to push atoms into a $1 \mathrm{D}$ narrow-line MOT. Because the atoms are polarized in magnetically trapped $m_{J}>0$ substates, they are confined by magnetic forces along $\pm x$, $\pm y$ and $+z$ and an optical force along $-z$. Cloud expansion measurements show asymmetric velocities corresponding to temperatures $T_{x}=(48 \pm 3.3) \mu \mathrm{K}$ along the $x$ direction and $T_{z}=(3.9 \pm 0.4) \mu \mathrm{K}$ along the $z$ direction,

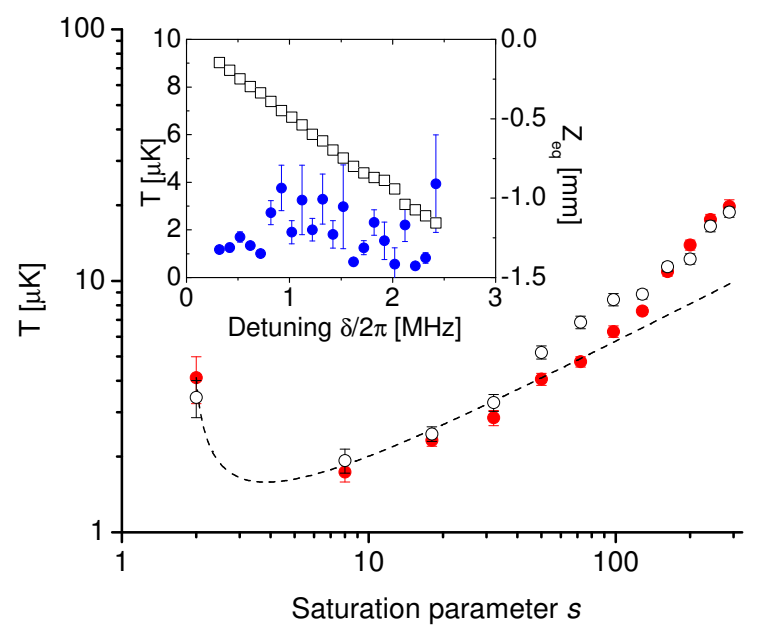

FIG. 4: (Color online) Narrow-line MOT temperatures $T_{x}$ (open) and $T_{z}$ (filled) as a function of the axial-beam saturation parameter $s$. The dashed curve is a fit to Eq. (2) for $s \leq 100$, with fit parameters $T_{D}=540 \mathrm{nK}$ and $\mathfrak{F}_{0}=0.65$, to be compared with the expectations $T_{D}=180 \mathrm{nK}$ and $\mathfrak{F}_{0}=0.3$. Inset: Temperature $T_{z}$ (circles, left axis) and trap position $z_{e q}$ (squares, right axis) vs. detuning $\delta / 2 \pi$.

indicating cooling along the direction of laser propagation. In image b), we used all three retroreflected beams. Here, we see cooling and confinement in all directions with more symmetric expansion velocities corresponding to $T_{x}=(4.8 \pm 2.1) \mu \mathrm{K}$ and $T_{z}=(1.2 \pm 0.3) \mu \mathrm{K}$.

We measured the temperature of the 3D narrow-line MOT as a function of $s$ and $\delta$, for a ratio of (total) transverse to axial beam power of 0.44. A number of predictions of the 1D model are confirmed in Fig. 4. In particular, the temperature exhibits a minimum for a small value of $s$ and increases as $\sqrt{s}$ for $s \leq 100$, the temperature is largely independent of $\delta$, and the trap position varies linearly with $\delta$. (Note the similar behavior in Refs. [5, [6] where red-detuned lasers balanced $\mathrm{Sr}$ atoms against gravity).

To test the spin polarization, we turn off the $841 \mathrm{~nm}$ lasers at $t=0$, leaving all magnetic fields on. Because atoms are in weak-field seeking states, they feel a restoring force toward the origin. As shown in Fig. [5, atoms begin oscillating in the conservative potential with a period of approximately $30 \mathrm{~ms}$. We observe high-fidelity oscillations in the position of peak density, with the oscillation period indicating a spin polarization of $(74 \pm 6) \%$. After a few oscillation periods, the anharmonic quadrupole potential leads to complicated cloud trajectories [20].

Our trap is relatively insensitive to the polarization of the transverse beams, and forms even when some retroreflections are blocked. In contrast, the trap is very sensitive to the polarization of the vertical trapping laser, while the retroreflection of this beam is inconsequential. 


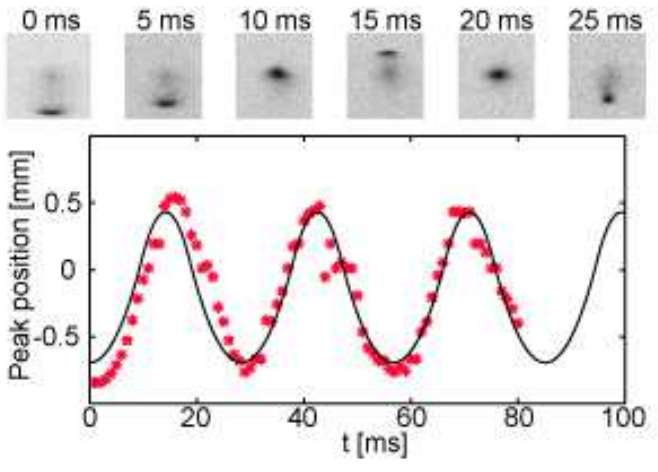

FIG. 5: (Color online) Oscillation of the atomic cloud after release into the magnetic trap. (Upper) Cloud images at early times. (Lower) $z$ position of maximum density together with a fit to the expected trajectory.

We have formed traps above and below the origin by reversing the direction of a single beam. The lower trap is more robust, presumably due to the stabilizing influence of gravity. These trap characteristics can be qualitatively explained from geometric considerations. Because of the large detuning and narrow atomic line, atoms approach resonance only within a narrow ellipsoid of constant magnetic field [5, 6]. $\pi$ and $\sigma^{-}$transitions can be neglected due to the large value of $\delta$, so the polarizations of the transverse beams only affect the coupling efficiency to the $\sigma^{+}$transition, via a position-dependent geometrical factor. Although the overall behavior of the trap is well-understood, our measured temperatures are always slightly higher than predicted by the simple 1D model. This discrepancy may be explained by heating during temperature measurement, since we observe that transients during magnetic field shutoff impart residual cloud velocities of a few $\mathrm{cm} / \mathrm{s}$. Given the total energy required to accelerate the cloud, a few percent spread in spin polarization results in a few percent spread in cloud velocity, corresponding to $\mu \mathrm{K}$-scale heating. The cloud temperature may also be affected by stimulated transitions or polarization-gradient effects, which cannot be properly analyzed without a 3D quantum mechanical model.

In summary, we have demonstrated a new type of narrow-line MOT for strongly magnetic atoms, in which optical and magnetic forces play equally important roles.
In future experiments, we plan to optimize loading procedures in order to study the atom number and phase-space density for this trap. These techniques should be applicable to the other rare-earth-metal elements and to alkaline earth elements in magnetically trapped, metastable states.

The authors gratefully acknowledge contributions from S. A. Lee. This work has been supported in part by the NIST-CNST/UMD-NanoCenter Cooperative Agreement. A. B. acknowledges financial support from the National Research Council.

[1] Y. Castin, H. Wallis, and J. Dalibard, J. Opt. Soc. Am. B 6, 2046 (1989).

[2] H. Wallis and W. Ertmer, J. Opt. Soc. Am. B 6, 2211 (1989).

[3] H. Katori et. al., Phys. Rev. Lett. 82, 1116 (1999).

[4] T. Mukaiyama et. al., Phys. Rev. Lett. 90, 113002 (2003).

[5] T. H. Loftus et. al., Phys. Rev. Lett. 93, 073003 (2004).

[6] T. H. Loftus et. al., Phys. Rev. A 70, 063413 (2004).

[7] S. B. Nagel et. al., Phys. Rev. Lett. 94, 083004 (2005).

[8] E. A. Curtis, C. W. Oates, and L. Hollberg, Phys. Rev. A 64,031403(R) (2001).

[9] T. Binnewies et. al., Phys. Rev. Lett. 87, 123002 (2001).

[10] J. Grünert and A. Hemmerich, Phys. Rev. A 65, 041401(R) (2002).

[11] N. Malossi et. al., Phys. Rev. A 72, 051403(R) (2005).

[12] H. Y. Ban et. al., Opt. Express 8, 3185 (2005).

[13] P. D. Lett et. al., J. Opt. Soc. Am. B 6, 2084 (1989).

[14] H. J. Metcalf and P. van der Straten, Laser Cooling and Trapping (Springer, New York, 1999).

[15] J. J. McClelland and J. L. Hanssen, Phys. Rev. Lett 96, 143005 (2006).

[16] A. J. Berglund, S. A. Lee, and J. J. McClelland, Phys. Rev. A 76, 053418 (2007).

[17] J. J. McClelland, Phys. Rev. A 73, 064502 (2006).

[18] R. W. Fox, C. W. Oates, and L. W. Hollberg, CavityEnhanced Spectroscopies: Experimental Methods in the Physical Sciences (Academic Press, 2002), chap. 1.

[19] Quoted errors and error bars represent one standard deviation combined random and systematic uncertainty.

[20] See EPAPS for a movie of the cloud oscillation for $100 \mathrm{~ms}$ after release into the magnetic trap. For more information on EPAPS, see http://www.aip.org/pubservs/epaps.html 